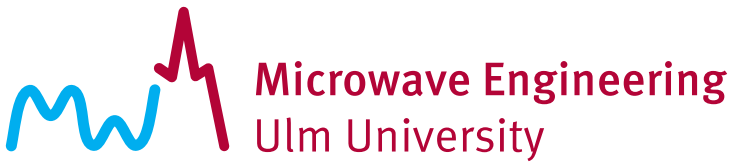

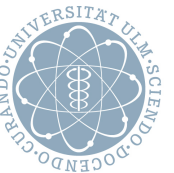

\section{Random Multiplexing for an MIMO-OFDM Radar With Compressed Sensing-Based Reconstruction}

Christina Knill, Fabian Roos, Benedikt Schweizer, Daniel Schindler, and Christian Waldschmidt

(C) 2019 IEEE. Personal use of this material is permitted. Permission from IEEE must be obtained for all other uses, in any current or future media, including reprinting/republishing this material for advertising or promotional purposes, creating new collective works, for resale or redistribution to servers or lists, or reuse of any copyrighted component of this work in other works. 


\title{
Random Multiplexing for an MIMO-OFDM Radar With Compressed Sensing-Based Reconstruction
}

\author{
Christina Knill, Student Member, IEEE, Fabian Roos, Student Member, IEEE, Benedikt Schweizer, Student \\ Member, IEEE, Daniel Schindler, and Christian Waldschmidt, Senior Member, IEEE
}

\begin{abstract}
In many applications, the direction of arrival (DoA) information of the radar signal plays a decisive role in target localization. A multiple-input multiple-output (MIMO) radar allows to obtain the position of an object in space within one measurement frame. Recent research and publications verify the high potential of digital radar principles such as orthogonal frequency-division multiplexing (OFDM). In this letter, a MIMOOFDM approach based on random frequency and time-division multiplexing is presented. It is enhanced by a multidimensional compressed sensing method which utilizes the information of multiple channels. The approach is validated and compared to other MIMO-OFDM approaches using measurements of an experimental radar at $72.5 \mathrm{GHz}$.
\end{abstract}

Index Terms-OFDM, MIMO, compressed sensing, radar, carrier interleaving, FDM, TDM

\section{INTRODUCTION}

$\mathbf{C}$ OMMON multiple-input multiple-output (MIMO) concepts for radar are mostly based on generating orthogonal signals for the different transmitters via time- or frequencydivision multiplexing (TDM or FDM). Usually, the assignment in time or frequency to these transmitters is according to a regular and periodic pattern such as alternating transmission. It applies to both TDM and FDM, solely one transmitter is active at a specific time or frequency.

Multicarrier waveforms such as orthogonal frequencydivision multiplexing (OFDM) receive increasing attention in the field of radar due to their high flexibility and alldigital signal generation. OFDM was first proposed for radar applications in [1] and has evolved continuously ever since. In contrast to conventional FMCW radars, OFDM offers the possibility of both TDM and FDM. In literature, there are only a few different MIMO-OFDM radar approaches, mostly FDM, including regular or equidistant interleaving [2], irregular or non-equidistant interleaving [3], and dynamic interleaving [4] (cf. Fig. 1(a)-(c)). The most common approach is the regular interleaving which uses subcarriers that are assigned equidistantly and fixed to the transmitters. However, this leads to regular spectral subsampling of each channel by a factor equal to the number of transmit antennas which again results in a reduced maximum unambiguous range

This work was supported by the Ministerium für Wissenschaft, Forschung und Kunst (MWK) Baden-Würrtemberg within the Tech Center a-drive under grant 32-7533-4-10/13/20.

C. Knill, F. Roos, B. Schweizer and C. Waldschmidt are with the Institute of Microwave Engineering, Ulm University, 89081 Ulm, Germany, e-mail: christina.knill@uni-ulm.de

D. Schindler is with the Corporate Research and Advance Engineering of Robert Bosch GmbH, 71272 Renningen, Germany compared to full OFDM. The irregular interleaving approach solves this issue by using non-equidistant subcarriers and thereby avoiding regular spectral subsampling. Though, this improvement comes at the expense of a distinct artifact ridge along the range cells of each target. In [3], it is proposed to apply compressed sensing (CS) to mitigate this effect, however, due to the constant allocation of subcarriers over time, channel information of only a single transmitter is available per subcarrier which is detrimental in CS signal reconstruction. In dynamic interleaving, the subcarriers are dynamically assigned to the transmitters over time. This preserves the unambiguity in all dimensions at the expense of a decreased SNR.

In this work, a random TDM/FDM-MIMO concept for OFDM derived from the dynamic approach is presented that offers improved flexibility of the waveform design and neither suffers from loss of unambiguity nor decrease of SNR. To enhance the proposed MIMO processing, the main contribution of this letter is a multidimensional CS post-processing that enables the recovery of the full processing gain of conventional OFDM despite of interleaving. It further allows exploiting the information of all channels within one CS instance and thereby improving the reconstruction, speeding up the processing, and reducing the computational complexity. In context of OFDM radar, CS has recently been proposed in various fields such as interference mitigation [5], bandwidth reduction [6], and MIMO [3]. Additionally, since both [3] and [4] lack a treatment of the directions of arrival (DoA), we verify that the DoA information is preserved.

\section{OFDM RADAR}

A conventional OFDM radar signal consists of a full frame yielding $N$ subcarriers, with subcarrier spacing $\Delta f$ and total bandwidth of $B=N \Delta f$, and $M$ consecutive OFDM symbols. In digital processing, this signal can be represented as a matrix $\boldsymbol{X}$ where the rows hold samples per subcarrier and the columns samples per OFDM symbol. Using Fourier transform based evaluation of the received frame, an inverse discrete Fourier transform (IDFT) of every OFDM symbol yields the range information and a consecutive discrete Fourier transform (DFT) of every subcarrier yields the velocity information of the channel. It is of particular importance that the 2D-(I)DFT processing is still valid even if some elements of the matrix are missing because they are equal to zero [6]. However, depending on the distribution structure of these zeros within the evaluated frame, the Fourier processing may lead to rising artifacts or artifact ridges in the range-velocity $(r-v)$ domain. 


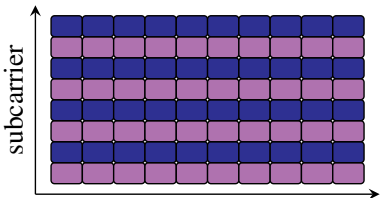

OFDM-symbol

(a) Regular interleaving.

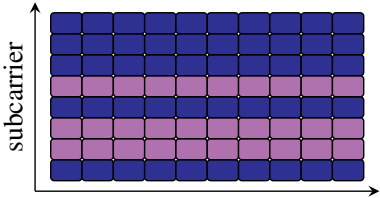

OFDM-symbol

(b) Irregular interleaving.

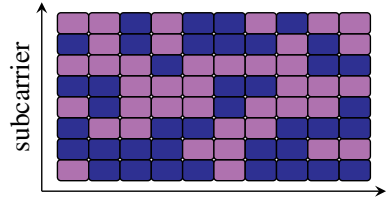

OFDM-symbol

(c) Dynamic interleaving.

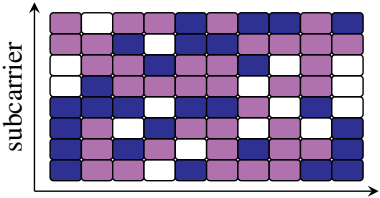

OFDM-symbol

(d) Random interleaving.

Fig. 1. Comparison of different MIMO-OFDM approaches in the frequency-time domain using two transmitters (Tx1: $\square$, Tx2: $\square$, unused: $\square$ ).

\section{RANDOM MIMO FOR OFDM}

By inserting additional steps in the conventional OFDM processing, an FDM or TDM multi-channel signal can be generated very easily based on a full frame OFDM $\boldsymbol{X}$. Using a distinct binary transmission matrix $\boldsymbol{\Phi}_{p}$, the signal of the $p$ th transmit antenna is determined by

$$
\boldsymbol{X}_{p}=\boldsymbol{X} \odot \boldsymbol{\Phi}_{p}, \quad \boldsymbol{\Phi}_{p} \in \mathbb{B}^{N \times M}
$$

where $\odot$ denotes the element-wise multiplication and the entry 1 in $\boldsymbol{\Phi}_{p}$ indicates active signal elements. In Fig. 1, the combined transmission patterns of regular, irregular, and dynamic interleaving are illustrated in (a) to (c), respectively. In (d), the proposed random MIMO approach is shown. Compared to (c) [4], more flexibility in the waveform design of the signals is facilitated since the only requirement is a quasi random pattern, individual elements may even be unused. Compared to (a) and (b) which are pure FDM transmission patterns, random MIMO is a combination of FDM and TDM. Considering a single subcarrier (row), it is a TDM scheme, considering a single OFDM symbol (column), it is an FDM scheme. The signal of the $p$ - $q$ th channel at receiver $q$ is extracted by

$$
\boldsymbol{Y}_{p, q}=\boldsymbol{Y}_{q} \odot \boldsymbol{\Phi}_{p} .
$$

The extraction of the $r-v$ map is carried out by 2D-(I)DFT according to the conventional OFDM. Since $\boldsymbol{\Phi}_{p}$ is sparse, this leads to a reduced processing gain of

$$
G_{p}=\sum_{n=0}^{N-1} \sum_{m=0}^{M-1}\left|\phi_{n, m}\right| \approx \frac{G_{\text {full }}}{P}
$$

compared to the processing gain $G_{\text {full }}$ of conventional OFDM where $P$ is the number of transmitters. Together with probably emerging artifacts, this leads to a degraded system dynamic. However, if the noise-free target information can be reconstructed for the missing samples, e.g., using CS, an increased artificial SNR is obtained and $G_{p}$ converges to $G_{\text {full }}$. In the following, the term SNR is used also for this artificial SNR and moreover encompasses system and artifact noise.

\section{Multi-Channel Compressed Sensing}

Since CS methods require random structures for optimal performance, they are well-suited for the given task of restoring missing signal samples and thereby regaining the full processing gain of the system. CS applied to SISO OFDM $r-v$ maps is presented in [6] where a single channel timefrequency OFDM signal is reconstructed from its patchworklike receive signal using iterative CS approaches. However, in MIMO applications, single channel CS would become more cumbersome and time-consuming with increasing number of channels. Therefore, an adaption and extension of the HAMP (hard threshold approximate message passing) approach from [6] is proposed in the following. It offers modified thresholding and signal estimation based on non-coherent integration (NCI) to utilize the intertwined information of multiple channels during reconstruction and thereby achieve estimation of all channels within one CS instance. This not only improves the reconstruction performance but also reduces its complexity. Additionally, the approach preserves the channel phase information during reconstruction which is crucial for reliable DoA estimation, as explained later.

For every channel, the received signal exhibits missing signal elements according to $\boldsymbol{\Phi}_{p}$. In the first step of the CS iteration, a 2D-(I)DFT is applied to each channel yielding their $r-v$ map. The second step is thresholding of these spectra. To avoid cumbersome thresholding of all channels, NCI of all channels is performed in advance which also combines knowledge from all channels. Afterwards, a hard threshold is applied to the combined 2D-matrix. Here, of interest are then not the threshold values but the locations of the selected elements with significant amplitudes. In the actual complexvalued spectra, only these elements are used to reconstruct the missing samples for each channel. Using a combined thresholding is of advantage for targets that are strong in most channels, but weak in some, since they might not pass a single-channel but a combined thresholding using multiple channels. In the third step, an estimate and residual of every channel in time-frequency domain is computed via inversion of the 2D-(I)DFT. Since this step is performed for each channel independently, the phase information of the targets is preserved and also restored in the reconstructed samples.

\section{Measurement Results}

For evaluation, an experimental OFDM radar with 2 transmitters and 4 receivers is used. The OFDM signal parameters are summarized in Table I. The virtual array of the setup has 10 elements with $\lambda / 2$ spacing where elements 2 and 9 are missing. Measurements were performed in an anechoic chamber using four corner reflectors placed at different angles. In Fig. 2a, the measurement setup along with the position of the targets is shown. The measured $r-v$ map (NCI of all channels) using the proposed MIMO approach with multichannel CS reconstruction is given in Fig. 2b. It shows that the four targets are clearly discernible. Applying simple DoA evaluation via Fourier transform of the channels results in the $x-y$ map presented in Fig. 2c. It confirms that the proposed CS reconstruction preserves the angle information of the targets. 


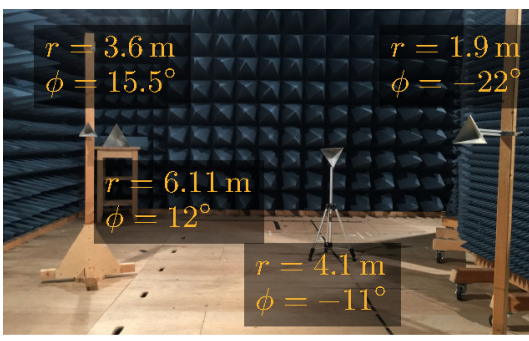

(a) Measurement setup in the anechoic chamber with four corner reflectors.

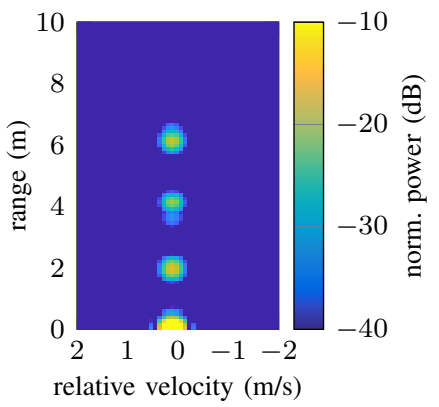

(b) NCI $r-v$ maps of the evaluation signals of all channels.

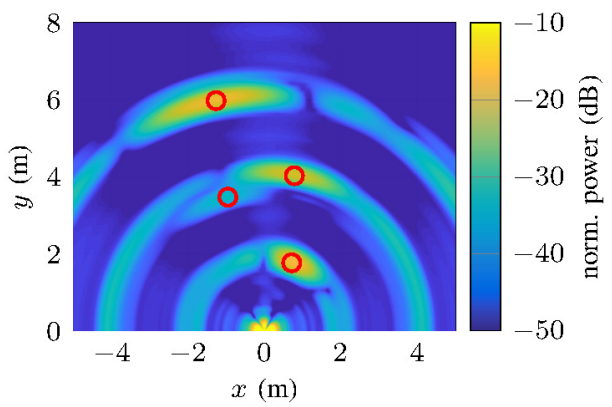

(c) Evaluated signal in $x-y$ domain after DoA evaluation (O: ground truth).

Fig. 2. Measurements: (a) The setup and its result in (b) $r-v$ and (c) $x-y$ domain. Peaks with range close to $0 \mathrm{~m}$ are caused by crosstalk between the antennas.

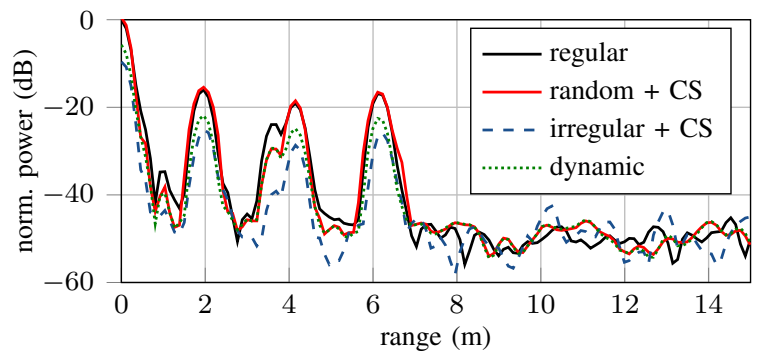

Fig. 3. Range profiles of measurements using the MIMO approaches regular, irregular (+ CS), and dynamic interleaved compared to the random approach with CS. Profiles are normalized to the noise floor of regular MIMO.

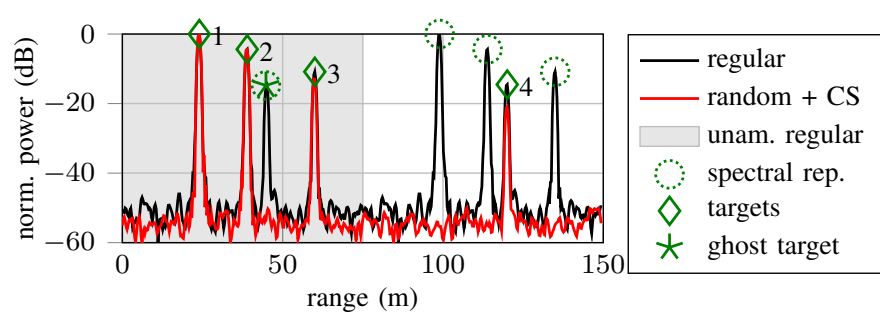

Fig. 4. Comparison of the simulated range profiles of regular and random interleaving using four targets $(\diamond)$. Since the unambiguous range of regular interleaving ( $\square$ ) is halved compared to random, spectral repetitions (ऋ) appear and targets outside this region fold back and appear as ghosts $(\star)$.

To compare the achievable performances of the different MIMO approaches, the obtained range profiles of the scene in Fig. 2a are shown in Fig. 3. For the random and the irregular approach, HAMP is applied before radar evaluation. Comparing the results, regular and random MIMO achieve similar results regarding SNR which is about 6 to $10 \mathrm{~dB}$ higher than that of dynamic and irregular. In case of regular, this

TABLE I

OFDM RADAR SIGNAL PROPERTIES

\begin{tabular}{c|c|c|c}
\hline Parameter & Symbol & Measurement & Simulation \\
\hline Number of symbols & $M$ & 3000 & 2048 \\
Number of subcarriers & $N$ & 2048 & 256 \\
Bandwidth (MHz) & $B$ & 650 & 256 \\
Subcarrier spacing (kHz) & $\Delta f$ & 317 & 1000 \\
\hline
\end{tabular}

is due to the lack of artifacts, in case of random MIMO, due to the additional processing gain achieved by CS. Using simulations with parameters given in Table I, the unambiguous range of regular and random MIMO is evaluated. The results of a scene where one target is intentionally placed outside the unambiguous range of regular MIMO but within that of full OFDM is presented in Fig. 4. Due to the decreased unambiguous range, a ghost target appears for regular but not for random MIMO.

\section{CONCLUSION}

In this letter a random MIMO approach for OFDM enhanced by a multidimensional CS method is presented which utilizes the intertwined information of all channels to restore efficiently missing signal samples. The processing is validated using measurements of an experimental OFDM radar. It is shown that the angle information of the channels is preserved during CS reconstruction. With the proposed approach, similar performance as with regular interleaving is achieved and, additionally, also the unambiguous range and velocity of full OFDM is preserved.

\section{ACKNOWLEDGMENT}

The authors would like to thank the Staff of Corporate Research and Advance Engineering, Robert Bosch GmbH, Stuttgart for their kind cooperation and provision of measuring equipment and data.

\section{REFERENCES}

[1] N. Levanon, "Multifrequency complementary phase-coded radar signal," IEE Proc. Radar, Sonar and Navigation, vol. 147, no. 6, pp. 276-284, 2000.

[2] C. Sturm et al., "Spectrally interleaved multi-carrier signals for radar network applications and multi-input multi-output radar," IET Radar, Sonar \& Navigation, vol. 7, no. 3, pp. 261-269, Mar. 2013.

[3] G. Hakobyan et al., "A Novel OFDM-MIMO Radar with Non-Equidistant Subcarrier Interleaving and Compressed Sensing," in Int. Radar Symp. (IRS), 2016.

[4] G. Hakobyan et al., "A Novel OFDM-MIMO Radar with Non-Equidistant Dynamic Subcarrier Interleaving," in Eur. Radar Conf. (EuRAD), 2016.

[5] B. Nuss et al., "A Novel Technique for Interference Mitigation in OFDM Radar Using Compressed Sensing," in IEEE MTT-S Int. Conf. on Microwaves for Intelligent Mobility (ICMIM), Mar. 2017, pp. 143-146.

[6] C. Knill et al., "High Range and Doppler Resolution by Application of Compressed Sensing Using Low Baseband Bandwidth OFDM Radar,' IEEE Trans. Microw. Theory Techn., vol. 66, no. 7, pp. 3535-3546, Jul. 2018. 